\title{
Evidence for an early evolutionary emergence of $\gamma$-type carbonic anhydrases as components of mitochondrial respiratory complex I
}

\author{
Ryan MR Gawryluk and Michael W Gray*
}

\begin{abstract}
Background: The complexity of mitochondrial complex I (Cl; NADH:ubiquinone oxidoreductase) has increased considerably relative to the homologous complex in bacteria. Comparative analyses of $\mathrm{Cl}$ composition in animals, fungi and land plants/green algae suggest that novel components of mitochondrial $\mathrm{Cl}$ include a set of 18 proteins common to all eukaryotes and a variable number of lineage-specific subunits. In plants and green algae, several purportedly plant-specific proteins homologous to $\gamma$-type carbonic anhydrases ( $\gamma(\mathrm{CA})$ have been identified as components of $\mathrm{Cl}$. However, relatively little is known about $\mathrm{Cl}$ composition in the unicellular protists, the characterizations of which are essential to our understanding of $\mathrm{Cl}$ evolution.

Results: We have performed a tandem mass spectrometric characterization of $\mathrm{Cl}$ from the amoeboid protozoon Acanthamoeba castellanii. Among the proteins identified were two YCA homologs, AcCa1 and AcCa2, demonstrating that $\gamma C A$ proteins are not specific to plants/green algae. In fact, through bioinformatics searches we detected $\gamma C A$ homologs in diverse protist lineages, and several of these homologs are predicted to possess $\mathrm{N}$-terminal mitochondrial targeting peptides.

Conclusions: The detection of $\gamma C A$ s in $\mathrm{Cl}$ of Acanthamoeba, considered to be a closer relative of animals and fungi than plants, suggests that $\mathrm{CCA}$ proteins may have been an ancestral feature of mitochondrial $\mathrm{Cl}$, rather than a novel, plant-specific addition. This assertion is supported by the presence of genes encoding $\gamma C A s$ in the nuclear genomes of a wide variety of eukaryotes. Together, these findings emphasize the importance of a phylogenetically broad characterization of $\mathrm{Cl}$ for elucidating $\mathrm{Cl}$ evolution in eukaryotes.
\end{abstract}

\section{Background}

Mitochondrial complex I (CI; NADH:ubiquinone oxidoreductase) is a multi-subunit and intricate proton pump that is responsible for the first step in the canonical respiratory chain - the oxidation of NADH and subsequent reduction of ubiquinone. Investigations of $\mathrm{CI}$ structure in animals and fungi portray $\mathrm{CI}$ as an L-shaped complex consisting of a hydrophobic domain integrated into the inner mitochondrial membrane and a hydrophilic mitochondrial matrix domain that oxidizes NADH and transfers electrons along 8-9 Fe-S clusters [1]. Mitochondrial CI comprises $35-45$ proteins $(\sim 900-1000 \mathrm{kDa})$,

\footnotetext{
*Correspondence: m.w.gray@dal.ca

1 Centre for Comparative Genomics and Evolutionary Bioinformatics, Department of Biochemistry and Molecular Biology, Dalhousie University, Halifax, Nova Scotia B3H 1X5, Canada

Full list of author information is available at the end of the article
}

which are encoded in both the mitochondrial and nuclear genomes. Conversely, the homologous $\sim 550-\mathrm{kDa}$ bacterial CI consists of only 14 subunits [2], suggesting a massive expansion of $\mathrm{CI}$ in eukaryotes.

The subunit composition of mitochondrial CI has been intensively studied in animals [3], fungi $[4,5]$ and land plants/green algae [6,7]. Comparative proteomic and genomic analyses of $\mathrm{CI}$ composition in these groups have provided insight into the evolution of mitochondrial CI: specifically, in all three groups, the 14-subunit 'bacterial core' (corresponding to the subunits inherited from the $\alpha$-proteobacterial ancestor of mitochondria; [8]) has been retained, while an additional 18 -subunit 'eukaryotic core' (made up of proteins shared by these eukaryotic lineages but not by bacterial CI) along with a variable number of lineage-specific proteins has been added [9]. Few of the lineage-specific proteins are similar to other proteins of 
known function in available databases; however, in plants $[7,10]$ and green algae [6], CI contains multiple proteins with high similarity to $\gamma$-type carbonic anhydrases $(\gamma \mathrm{CAs})$, first described as a homotrimeric complex (Cam) in the methanogenic archaeon, Methanosarcina thermophila [11].

Carbonic anhydrases are ubiquitous metalloenzymes that catalyze the reversible hydration of $\mathrm{CO}_{2}$ to $\mathrm{HCO}_{3}$. Five evolutionarily unrelated CA classes $(\alpha, \beta, \gamma, \delta$, and $\zeta)$ are currently known, suggesting that this important enzymatic mechanism has been invented independently multiple times. The $\gamma \mathrm{CA}$ class is among the most ancient, with homologs widespread in Archaea and Bacteria [12]; however, in eukaryotes, $\gamma \mathrm{CA}$ homologs have been described from a phylogenetically narrow collection of species, comprising almost exclusively green algae and land plants, in association with CI. In this group, singleparticle electron microscopy studies have demonstrated the presence of an additional CI domain [13,14]. This novel structure, likely representing the $\gamma \mathrm{CA}$ proteins, is associated with the inner membrane portion of $\mathrm{CI}$ and projects into the mitochondrial matrix. The function of $\gamma \mathrm{CA}$ proteins in $\mathrm{CI}$ remains enigmatic: thus far, carbonic anhydrase activity has not been detected biochemically. However, experiments in Arabidopsis have demonstrated that expression of plant $\gamma \mathrm{CAs}$ is affected by $\mathrm{CO}_{2}$ concentration (see [15]) and that plant $\gamma C A$ proteins bind inorganic carbon [16]. These functional observations, coupled to the apparent restriction of $\gamma C A$ s to mitochondria of plant/green algal lineages, have prompted the proposal that CI $\gamma \mathrm{CA}$ proteins may have plant-specific functions; for instance, Braun \& Zabaleta [17] have suggested that $\gamma C A$ proteins play an important role in chloroplast function via the generation of $\mathrm{HCO}_{3}{ }^{-}$in the recycling of inorganic carbon for $\mathrm{CO}_{2}$ fixation.

Although $\gamma \mathrm{CA}$ proteins are not components of $\mathrm{CI}$ in animals and fungi (see [9]), little is known about the composition of $\mathrm{CI}$ in the predominantly unicellular protists. Because protists comprise the bulk of eukaryotic diversity, it is important to characterize CI from diverse lineages in order to understand fully the evolution of this protein complex. In particular, we cannot conclude that supposedly lineage-specific CI subunits are truly lineagespecific without knowledge of CI composition in the majority of eukaryotes. To this end, we have isolated an enzymatically active, $\sim 940-\mathrm{kDa}$ native $\mathrm{CI}$ and an inactive, $\sim 820-\mathrm{kDa}$ CI subcomplex $\left(\mathrm{CI}^{*}\right)$ from the amoeboid protist Acanthamoeba castellanii via blue native polyacrylamide gel electrophoresis (BN-PAGE) and have carried out a characterization of subunit composition via tandem mass spectrometry (MS/MS). Here we describe the detection of two $\gamma \mathrm{CA}$ homologs, $\mathrm{AcCa}$ a and $\mathrm{AcCa} 2$, in both forms of Acanthamoeba CI. Acanthamoeba is a member of the eukaryotic supergroup Amoebozoa and is not a close relative of plants [18]; rather, available evidence indicates that Amoebozoa comprises the evolutionary sister group to the opisthokonts (animals, fungi and their specific unicellular relatives). The unexpected finding of $\gamma \mathrm{CA}$ homologs in mitochondrial CI of Acanthamoeba demonstrates that these proteins are not limited to CI of plants/green algae, as was previously suggested [10]. In fact, we provide evidence here that $\gamma C A$ homologs having predicted mitochondrial targeting peptides (mTPs) are widespread throughout the domain Eucarya.

\section{Results and Discussion $\gamma$ Carbonic anhydrase proteins in Acanthamoeba $\mathrm{Cl}$}

An in-gel assay for NADH dehydrogenase activity was performed on Acanthamoeba mitochondrial protein complexes separated by BN-PAGE, yielding an insoluble formazan precipitate at $\sim 940 \mathrm{kDa}$ (Figure 1A). This molecular weight estimate is in agreement with the findings of Navet et al. [19] and corresponds to the general size of CI across eukaryotes. Together, these observations suggest that the identified NADH dehydrogenase activity corresponds to the native, enzymatically active CI of Acanthamoeba. Furthermore, analysis of Acanthamoeba mitochondrial protein complexes by two-dimensional BN/SDS-PAGE revealed another, more abundant com-

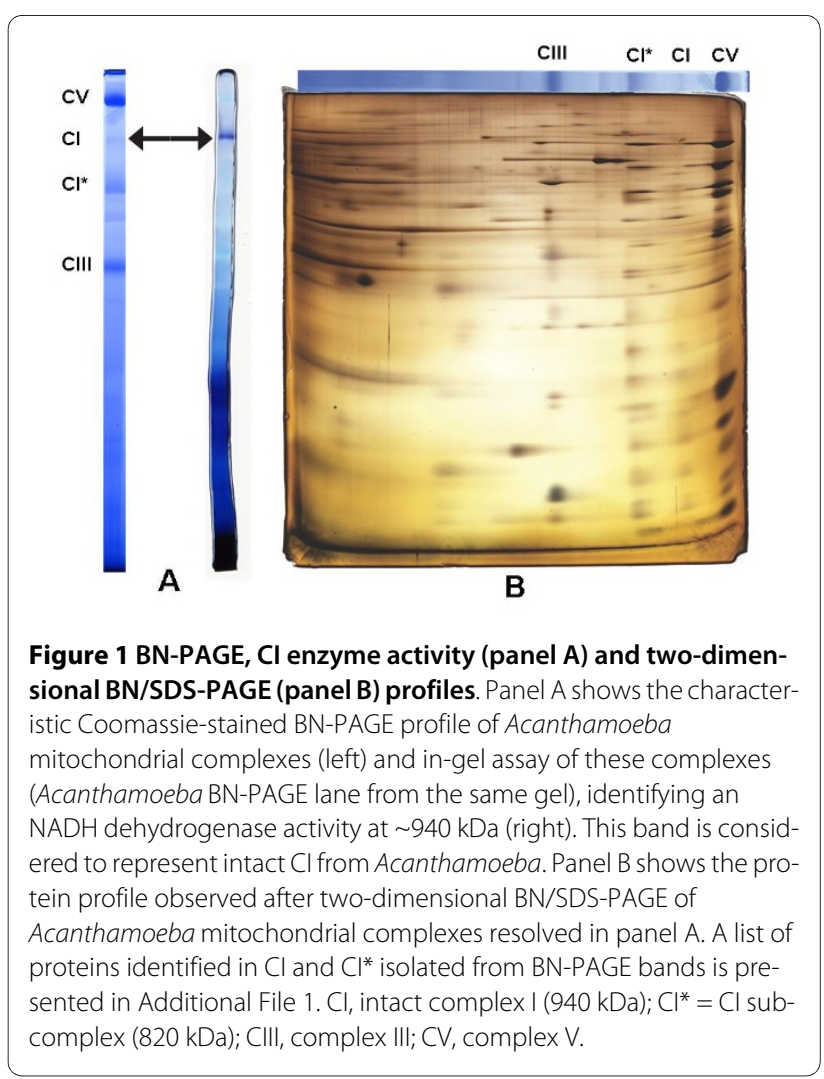


plex $\left(\mathrm{CI}^{*}\right)$ of $\sim 820 \mathrm{kDa}$ that has a very similar protein profile to intact CI (Figure 1B). Based on the twodimensional BN/SDS-PAGE profile and lack of enzyme activity following one-dimensional BN-PAGE, we infer that this complex is likely a partially dissociated CI missing a portion of the NADH-oxidizing mitochondrial matrix domain. The dissociation of $\mathrm{CI}$ into subcomplexes during BN-PAGE is not uncommon, and the observed breakdown into a slightly smaller, enzymatically inactive subcomplex is reminiscent of the situation in the green alga Polytomella [14].

Tandem mass spectrometric analysis of the $940-\mathrm{kDa}$ and $820-\mathrm{kDa}$ bands demonstrated that both were enriched in CI subunits known from other organisms, supporting our conclusion that both represent CI (see Additional File 1). A number of contaminating, highabundance mitochondrial proteins from other complexes were also present; this is not unexpected, as the relative abundance of $\mathrm{CI}$ is low in comparison with other complexes, and $\mathrm{CI}^{*}$ appears to co-migrate with other abundant complexes (Figure 1B). Notably, two $\gamma \mathrm{CA}$ homologs were detected in both the $940-\mathrm{kDa}$ and $820-\mathrm{kDa}$ samples. These proteins, here named AcCa1 and AcCa2, are 36\% identical to each other and are the only $\gamma C A$ homologs represented in the Acanthamoeba TBestDB EST library [20] and the ongoing Acanthamoeba nuclear genome project (Baylor College of Medicine; http:// www.hgsc.bcm.tmc.edu/microbial-

detail.xsp?project id=163). Between the two samples analyzed by MS/MS, $31 \%$ and $47 \%$ of the AcCa1 and AcCa2 protein sequences were covered, respectively (see Additional File 2). These findings constitute the first report of $\gamma \mathrm{CA}$ homologs as $\mathrm{CI}$ components outside of the plant/green algal lineage (see Figure 2 for multiple alignment). Based on these results, we suggest that AcCa1 and $\mathrm{AcCa} 2$ are matrix-facing components of the inner membrane arm of CI in Acanthamoeba, as are their homologs in plants/green algae [13,14].

Because not all expected CI subunits were detected in the MS/MS analysis and because the main contaminants of our CI proteins were ATP synthase proteins, we considered the possibility that the $\gamma C A$ proteins might actually be ATP synthase $(\mathrm{CV})$ subunits. The enzymatically active CI complex is present in very low abundance in $A$. castellanii, so that detection of all CI subunits is proving to be a challenge. Conversely, CV is extraordinarily abundant, as is evident in Figures 1a and 1b; as a result, MS/ MS data have revealed all CV subunits in A. castellanii, whereas no $\gamma C A$ proteins were detected in this complex. These considerations combined with the fact that $\gamma \mathrm{CA}$ proteins have been characterized as bona fide CI components in other organisms effectively eliminates the possibility that they might be $\mathrm{CV}$ subunits instead in Acanthamoeba.
A small number of $\gamma C A$ homologs have been described in eukaryotes outside of the plant supergroup. A cDNA encoding a $\gamma C A$ homolog from the haptophyte alga Emiliania huxleyi was identified previously [21]; however the authors did not investigate the subcellular localization of the protein. We have previously identified three different $\gamma \mathrm{CA}$ homologs in a proteomic investigation of highly purified mitochondria from the cilated protozoon, Tetrahymena thermophila [22]. Interestingly, two of these three $\gamma C A$ homologs were detected in mass spectrometric analyses of a $1 \mathrm{MDa}$ aggregate from Tetrahymena mitochondrial membrane proteins separated by $\mathrm{BN}$ PAGE (Gawryluk et al., unpublished results). This aggregate is highly enriched in known protein components of complexes I, III and V, suggesting that the identified $\gamma \mathrm{CA}$ homologs may be components of CI in Tetrahymena as well.

From an evolutionary perspective, our findings are somewhat surprising: the Amoebozoa supergroup (to which Acanthamoeba belongs) is thought to be sister to the opisthokonts (animals + fungi), and is not closely related to the Plantae supergroup [18]. Since no authentic $\gamma \mathrm{CA}$ homologs were detected in opisthokont nuclear genome or EST sequence databases (and no $\gamma \mathrm{CA}$ homologs are part of opisthokont $\mathrm{CI}$ ), these results suggest that $\gamma C A$ proteins may have been specifically lost from opisthokont $\mathrm{CI}$, rather than recently added to the plant/green algal lineage. Moreover, the finding that $\gamma \mathrm{CA}$ homologs may be components of $\mathrm{CI}$ in other eukaryotic groups (ciliates) raises the possibility that $\gamma \mathrm{CA}$ homologs were an ancestral feature of mitochondrial CI (i.e., a component of the eukaryotic core of CI lost in animals and fungi). We expected that if this were the case, $\gamma \mathrm{CA}$ homologs should be detected in other protist lineages.

\section{ү Carbonic anhydrase proteins are found throughout Eucarya}

In order to better understand the distribution and evolution of $\gamma C A$ homologs across the domain Eucarya, we employed a systematic bioinformatics approach to search nuclear genomic and EST sequence databases from diverse eukaryotes. In contrast to previous reports that $\gamma C A$ proteins are largely limited to plants/green algae, we detected (often multiple) homologs in the majority of eukaryotic groups (Table 1; see also Additional Files 3, 4 and 5). $\gamma \mathrm{CA}$ homologs are found in other major groups within the Plantae supergroup, including one in the red alga Cyanidioschyzon merolae and at least two in the glaucophyte alga, Cyanophora paradoxa. Aside from the opisthokonts, homologs could be detected in most eukaryotic supergroups, including Amoebozoa (Acanthamoeba, Dictyostelium, Hartmannella, Hyperamoeba, Polysphondylium), Chromalveolata (Tetrahymena, Phytophthora, Phaeodactylum, Blastocystis, Emiliania, Guil- 


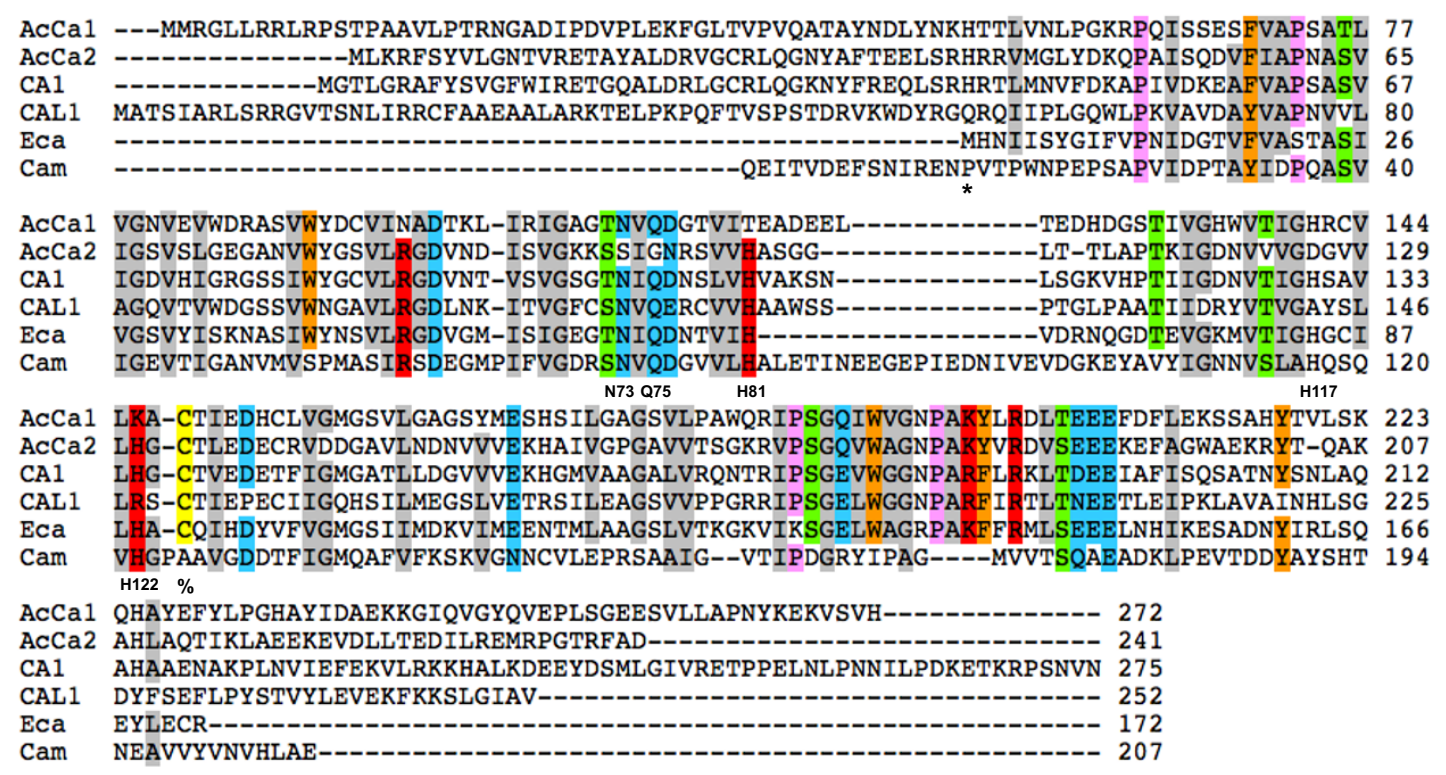

Figure 2 Alignment of AcCa1 and AcCa2 sequences with homologs from Arabidopsis and prokaryotes. Acanthamoeba AcCa1 and AcCa2 are aligned with Arabidopsis CA1 and CAL1 along with an a-proteobacterial (Ehrlichia canis) YCA homolog and archaeal Cam (Methanosarcina thermophila). Critical amino acids described in text are referred to according to the Cam nomenclature. H42 and C137 of CA1 are denoted by * and \%, respectively. Shading of columns reflects amino acid similarity of $\geq 80 \%$.

lardia), Excavata (Reclinomonas, Seculamonas, Naegleria, Euglena, Trypanosoma, Malawimonas) and possibly Rhizaria (an EST homolog was idenitified in Bigelowiella natans; however, several stop codons interrupt the reading frame, suggesting that the EST in question may represent an incompletely spliced transcript).

Of the retrieved $\gamma \mathrm{CA}$ protein sequences considered to be complete at their N-termini (where mTPs would be located; [23]), many are predicted to be mitochondrial by the programs TargetP [24] and MitoProtII [25] above a confidence level of $70 \%$, suggesting that $\gamma \mathrm{CA}$ proteins may be targeted to mitochondria in a wide variety of eukaryotes (see Additional File 6). Notably, several of the known mitochondrial $\gamma \mathrm{CA}$ proteins are not predicted to have mTPs with a given program (e.g. CA3 and CAL2 from Arabidopsis, AcCa2 from Acanthamoeba and all three $\gamma$ CAs from Tetrahymena with TargetP), so the lack of an apparent mTP does not preclude a mitochondrial localization. Genes encoding $\gamma \mathrm{CA}$ proteins are absent from the nuclear genome sequences of several eukaryotes that do not have conventional mitochondria (e.g. Trichomonas, Giardia) and are also absent from at least one eukaryote (Plasmodium) that specifically lacks CI, further suggesting that $\gamma \mathrm{CA}$ proteins may be targeted to mitochondria - and likely to CI - in most eukaryotes. Surprisingly, a $\gamma \mathrm{CA}$ homolog was detected in the nuclear genome of Entamoeba histolytica [26], an amoebozoon that does not possess conventional mitochondria (i.e., it lacks an electron transport chain). This homolog, however, does not appear to be closely related to other eukaryotic $\gamma C A s$, and may represent a lateral gene transfer from bacteria (data not shown).

To assess evolutionary relationships, we performed a phylogenetic analysis of eukaryotic and bacterial $\gamma \mathrm{CA}$ proteins, using the archaeal Cam sequence as outgroup. In the resulting phylogenetic trees (not shown), virtually all eukaryotic $\gamma C A$ proteins formed a large clade branching as a sister group to $\alpha$-Proteobacteria (the B. natans sequence branched within the latter clade whereas the $E$. histolytica sequence mentioned above, as well as a $G$. theta one, did not affiliate with the other eukaryotic $\gamma \mathrm{CA}$ sequences, but neither did they branch robustly with any particular bacterial clade). However, these trees were poorly resolved, with very little statistical (bootstrap) support for most partitions, so the phylogenetic conclusions that can be drawn from them are limited. Although the available data (Table 1 ) suggest an early $\gamma C A$ gene duplication during eukaryotic evolution, phylogenetic analysis was not able to delineate the point at which this may have occurred. We can infer, however, that the duplication predated the divergence of the amoebozoan taxa investigated here; moreover, we are able to identify the amoebozoan sequences that are orthologous with either AcCa1 or AcCa2 (Additional File 5). 
Table 1: Distribution of үCAs Throughout Eukaryotes.

\begin{tabular}{|c|c|c|c|c|}
\hline Supergroup & Taxon & YCA homolog? & Number of yCA homologs & \\
\hline \multirow[t]{4}{*}{ Plantae } & Arabidopsis & Yes & 5 & All are $\mathrm{Cl}$ subunits \\
\hline & Chlamydomonas & Yes & 3 & All are $\mathrm{Cl}$ subunits \\
\hline & Cyanidioschyzon & Yes & 1 & \\
\hline & Cyanophora & Yes & $\geq 2$ & \\
\hline \multirow[t]{5}{*}{ Amoebozoa } & Acanthamoeba & Yes & 2 & Both are $\mathrm{Cl}$ subunits \\
\hline & Dictyostelium & Yes & 2 & \\
\hline & Hartmannella & Yes & $\geq 1$ & \\
\hline & Entamoeba & Yes & 1 & No ETC \\
\hline & Polysphondylium & Yes & 2 & \\
\hline \multirow[t]{7}{*}{ Opisthokonta } & Saccharomyces & No & 0 & \\
\hline & Neurospora & No & 0 & \\
\hline & Ustilago & No & 0 & \\
\hline & Yarrowia & No & 0 & \\
\hline & Bos & No & 0 & \\
\hline & Drosophila & No & 0 & \\
\hline & Monosiga & No & 0 & \\
\hline \multirow[t]{7}{*}{ Chromalveolata } & Tetrahymena & Yes & 3 & All are mitochondrial \\
\hline & Plasmodium & No & 0 & Lacks Cl \\
\hline & Phytophthora & Yes & 2 & \\
\hline & Phaeodactylum & Yes & $\geq 2$ & \\
\hline & Blastocystis & Yes & $\geq 1$ & \\
\hline & Guillardia & Yes & $\geq 3$ & \\
\hline & Emiliania & Yes & $\geq 1$ & \\
\hline
\end{tabular}


Table 1: Distribution of $\gamma C$ As Throughout Eukaryotes. (Continued)

\begin{tabular}{llll}
\hline Rhizaria & Bigelowiella & Yes & $\geq 1$ \\
\hline Excavata & Reclinomonas & Yes & 2 \\
\hline Naegleria & Yes & $\geq 1$ \\
\hline Euglena & Yes & 2 & $\geq 2$ \\
\hline Trypanosoma & Yes & Yes & 0 \\
\hline Malawimonas & No & Lacks ETC
\end{tabular}

Organisms are arranged according to supergroup membership [18]. 'Number of $\gamma C A$ homologs' refers to the number of distinct $\gamma C A$ proteins encoded by an organism as determined by searches of EST or nuclear genomic databases. Inferred protein sequences are available in Additional Files 3 and 4

\section{Analysis of primary protein structure of $\gamma C A$ homologs} As earlier noted, plant and green algal $\gamma C A$ proteins constitute a CI domain that is associated with the matrix face of the inner membrane-integrated arm [13,14]. Arabidopsis encodes five $\gamma \mathrm{CA}$ homologs (CA1, CA2, CA3, CAL1 and CAL2), all of which interact with CI [27], while Chlamydomonas CI contains three different $\gamma \mathrm{CA}$ homologs [6]. Although Arabidopsis $\gamma C A 1-3$ (but not CAL1, CAL2) have retained nearly all residues known to be structurally or functionally important in the archetypal $\gamma \mathrm{CA}$ (Cam) from the methanogenic archaeon Methanosarcina thermophila [11], including the $\mathrm{Zn}^{2+}$ binding residues $\mathrm{H} 81$, H117 and H122 [10], the function of $\gamma C A$ homologs in plant mitochondria is still largely unknown. Plant $\gamma C A$ proteins lack two Glu residues (D61, D84) critical to the proton transfer mechanism of Cam and all previous attempts to demonstrate carbonic anhydrase activity in Arabidopsis mitochondrial extracts and in sucrose gradient-enriched plant CI [15] and recombinant $\gamma \mathrm{CA} 2$ homotrimers [16] have been unsuccessful. Like the plant homologs, Acanthamoeba $\gamma$ CAs lack D61 and D84; moreover, Acanthamoeba $\gamma$ CAs conserve fewer of the other residues critical to Cam function than do plant $\gamma \mathrm{CAs}$. In terms of $\mathrm{Zn}^{2+-}$ binding His residues, AcCa1 conserves only $\mathrm{H} 117$ whereas AcCa2 retains $\mathrm{H} 81$ and $\mathrm{H} 122$ (Figure 2). However, it should be noted that the three His residues required to bind $\mathrm{Zn}^{2+}$ in Cam are not localized within the same $\gamma C A$ monomer: rather, $\mathrm{Zn}^{2+}$ is coordinated by $\mathrm{H} 81$ and $\mathrm{H} 122$ of one monomer and $\mathrm{H} 117$ of a second monomer within the trimeric Cam structure [28]. It is noteworthy that the three His residues are distrib- uted between the two $\gamma \mathrm{CA}$ homologs found in Acanthamoeba, so it may be that the latter are able to bind $\mathrm{Zn}^{2+}$ in concert. However, in AcCa2, S and G replace N73 and Q75, respectively; in Cam, N73 and Q75 are proposed to be critical components of the catalytic mechanism [28]. Taken together, these findings suggest that the $\gamma \mathrm{CA}$ homologs of Acanthamoeba CI may not possess a carbonic anhydrase activity. On the other hand, carbonic anhydrase activity has been reported for a comparably divergent, recombinant $\gamma \mathrm{CA}$ from the haptophyte Emiliania huxleyi [21].

As is the case with the Acanthamoeba proteins, the vast majority of $\gamma C A$ proteins from eukaryotes outside of the plant supergroup do not individually conserve all three metal-binding His residues, although all three are often found among different $\gamma \mathrm{CAs}$ where multiple isoforms are present. Thus, it is still unclear whether carbonic anhydrase activity or $\mathrm{Zn}^{2+}$ binding is likely to be a general feature of $\gamma C A s$ across eukaryotes. Interestingly, several other positions in our phylogenetically broad multiple alignment are fairly well conserved across eukaryotes (and in some cases, bacteria), but not in Cam; it is possible that the conservation of these residues points to novel, potentially important functional sites. For instance, a His residue (corresponding to $\mathrm{H} 42$ of Arabidopsis $\gamma \mathrm{CA} 1$ ) is present in 27 of the 40 eukaryotic homologs with complete $\mathrm{N}$-terminal sequence that we identified in this study, but not in bacterial/archaeal $\gamma \mathrm{CA}$ homologs, while a Cys residue (Arabidopsis $\gamma \mathrm{CA} 1 \mathrm{C137})$ is highly conserved in eukaryotes and bacteria (41/48), but not in Cam (see Additional File 4). Additionally, many 
of the eukaryotic $\gamma \mathrm{CA}$ homologs (including the Acanthamoeba ones) have $\mathrm{C}$-terminal extensions relative to their prokaryotic homologs. In land plants, these extensions have been implicated in integration into the inner mitochondrial membrane [14], further supporting the idea that $\gamma C A$ proteins may be components of $\mathrm{CI}$ in many diverse eukaryotes.

Interestingly, inspection of the $\mathrm{N}$-terminal portion of $\gamma \mathrm{CAs}$ from diverse eukaryotes demonstrates that there may be, in general, at least two distinct classes of $\gamma \mathrm{CA}$ proteins. In particular, the N-terminal $\sim 60$ amino acids of AcCa2, along with various protist $\gamma \mathrm{CAs}$, are highly similar to the corresponding region of Arabidopsis CA1-3, whereas the N-termini of AcCa1 and Arabidopsis CAL12 are not highly conserved (Figure 3 ). This observation suggests that there may be distinct roles or sub-localizations of the two classes of $\gamma \mathrm{CA}$ within $\mathrm{CI}$; specifically, the high degree of conservation of the $\mathrm{N}$-terminal region of the AcCa2/CA1-3 class, but not AcCa1/CAL1-2 class, implies that the functional constraints at protein $\mathrm{N}$-termini are stronger in the former class.

\section{Possible function(s) of $\gamma \mathrm{CA}$ proteins in $\mathrm{Cl}$}

Although in silico reconstructions of Arabidopsis $\gamma \mathrm{CA}$ proteins and comparisons to Cam suggest the possibility of carbonic anhydrase activity, the biochemical function of $\gamma \mathrm{CA}$ homologs in plant/green algal $\mathrm{CI}$ is controversial as there are currently no experimental data confirming that plant/green algal $\gamma C A$ proteins are carbonic anhydrases. Recently, however, it has been shown that Arabidopsis $\gamma \mathrm{CA} 2$ trimers are capable of binding inorganic carbon [16], and microarray studies indicate that expression of Arabidopsis $\gamma \mathrm{CA} 1$ and $\gamma \mathrm{CA} 2$ is down-regulated under high $\mathrm{CO}_{2}$ concentrations (see [15]). These results suggest that $\gamma C A$ homologs might play an important role in the metabolism or transport of one-carbon com- pounds in land plants. Although these observations provide evidence that $\gamma C$ As function in relation to inorganic carbon metabolism in plants, it is still unclear whether these observations can be generalized across eukaryotes. As discussed above, most eukaryotes do not conserve all three His residues required for $\mathrm{Zn}^{2+}$ coordination (and therefore binding of $\mathrm{CO}_{2}$ and $\mathrm{HCO}_{3}{ }^{-}$). Consequently, it may be that the function of $\gamma \mathrm{CA}$ proteins is somewhat different in plants and other eukaryotes, and that $\mathrm{CI} \gamma \mathrm{CA}$ proteins in non-plant species serve a predominantly structural role in the complex. Ultimately, in order to distinguish between these possibilities, it will be important to determine whether or not non-plant $\gamma C A$ proteins are capable of binding $\mathrm{Zn}^{2+}$.

Because $\mathrm{CI} \gamma \mathrm{CA}$ proteins were believed to be plant-specific, it has been proposed that they are involved in plantspecific processes, namely $\mathrm{HCO}_{3}$ - formation (and possibly $\mathrm{HCO}_{3}{ }^{-}$transport across the inner mitochondrial membrane), ultimately for $\mathrm{CO}_{2}$ fixation in chloroplasts [17]. Although we cannot rule out the possibility that CI $\gamma$ CAs play an active role in chloroplast metabolism in plants, the presence of $\gamma \mathrm{CA}$ homologs in CI of ancestrally aplastidic eukaryotes (e.g. Acanthamoeba) demonstrates that this could not have been the ancestral role of $\gamma C A$ s in $\mathrm{CI}$, and that these proteins must perform some role that is not related to chloroplast function. Other data suggest that $\gamma \mathrm{CA}$ proteins in plants may play a role in expression and assembly of mitochondrial CI. For instance, it was demonstrated that total levels of $\mathrm{CI}$, along with abundance of $\mathrm{CI}+\mathrm{CIII}$ supercomplexes, are dramatically decreased $(>80 \%)$ in Arabidopsis $\gamma C A 2$ and $\gamma C A 3$ knockout lines grown in suspension culture [15]. A reduction in total CI protein was detected (i.e., not just aberrant CI assembly), suggesting that $\gamma C A$ proteins play an important role in the expression/stability of CI subunits.

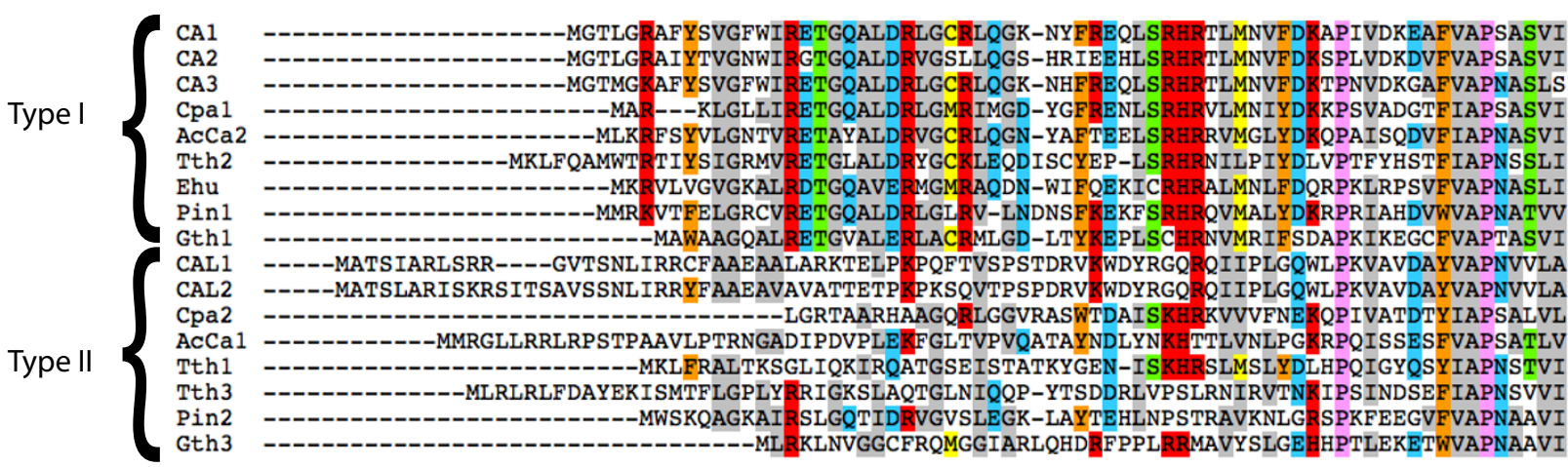

Figure 3 Distinct subtypes of eukaryotic $\gamma$ CAs. A truncated alignment of $\gamma C A$ proteins demonstrates that the $\mathrm{N}$-terminal regions of certain isoforms (termed Class I) are highly conserved throughout Eucarya (see text). Species abbreviations: CA1-3, Arabidopsis; Cpa, Cyanophora paradoxa; AcCa, Acanthamoeba castellanii; Tth, Tetrahymena thermophila; Ehu, Emiliania huxleyi; Pin, Phytophthora infestans; Gth, Guillardia theta. Shading of columns reflects amino acid similarity of $\geq 40 \%$. 
Recently, Tripp et al. [29] demonstrated that the active site of Cam contains $\mathrm{Fe}^{2+}$ instead of $\mathrm{Zn}^{2+}$ when the enzyme is reconstituted under anaerobic conditions. As noted by Parisi et al. [10], CI (along with the rest of the respiratory chain) contains multiple iron centers, so the Fe-binding ability of Cam may point to a role for $\gamma \mathrm{CA}$ proteins in mitochondrial respiratory control. Notably, Arabidopsis $\gamma \mathrm{CA} 2$, a physical component of CI, has been annotated as a transcription factor involved in the anther-specific expression of nucleus-encoded CI proteins (see GenBank accession no. AAK28403). The prospect that $\gamma C A$ proteins may function as CI-specific transcription factors is quite intriguing, as it would suggest a novel mechanism of communication between the mitochondrial electron transport chain and the nucleus, and would indicate a function for $\mathrm{CI} \gamma \mathrm{CA}$ proteins that could be applicable across all aerobic CI-containing eukaryotes, and not just photosynthetic ones.

\section{Conclusions}

The number of subunits comprising $\mathrm{CI}$ in eukaryotes has expanded markedly relative to the homologous bacterial complex. Previous comparative genomic analyses have suggested that 18 proteins were added to CI very early in eukaryotic evolution, while a smaller proportion are specific to particular lineages within the eukaryotic domain [9]. Although a number of the novel, 'lineage-specific' proteins are likely to be encoded by a phylogenetically restricted group of related organisms, others are currently deemed to be 'lineage-specific' only because our knowledge of CI composition across the eukaryotes is incomplete. We have detected two $\gamma C A$ homologs - previously thought to be plant/green algal CI proteins - as components of CI in an amoeboid protozoon, Acanthamoeba castellanii, an organism not specifically related to the plant/green algal lineage. The most parsimonious interpretation of our observations is that $\gamma C A$ proteins were part of the eukaryotic core of CI that was added early in eukaryotic evolution, and that they were subsequently lost in certain groups, most notably the opisthokonts. Moreover, $\gamma \mathrm{CA}$ proteins are known to be mitochondrial in ciliates (and may be CI proteins as well) and bioinformatics searches have revealed a large repertoire of $\gamma C A$ homologs in other major eukaryotic groups. These observations underscore the importance of characterizing the composition of mitochondrial protein complexes from a wide variety of organisms in order to understand fully and accurately their function and evolution.

\section{Methods}

\section{Cell Growth and Isolation of Mitochondria}

Mitochondria were prepared from two 500-ml cultures of Acanthamoeba castellanii (strain Neff) cells grown to an
$\mathrm{OD}_{580}$ of $\sim 1.0$, essentially as reported by Lohan and Gray [30]. The present method differed in that cells were not washed with phosphate-buffered saline prior to lysis, the crude mitochondrial pellet was washed only once with mitochondrial wash buffer $(10 \mathrm{mM}$ Tris. $\mathrm{HCl}, 10 \mathrm{mM}$ $\mathrm{Na}_{2}$ EDTA, $0.35 \mathrm{M}$ sucrose, $1 \mathrm{mM}$ dithiothreitol and $0.1 \%$ BSA) and mitochondria were purified in a SW27Ti rotor centrifuged at 22,000 $\mathrm{rpm}$. The protein concentration was assayed with the BioRad $D_{C}$ Protein Assay kit and whole mitochondria were diluted to a final protein concentration of $25 \mathrm{mg} / \mathrm{ml}$.

\section{Blue Native Polyacrylamide Gel Electrophoresis (BN-PAGE)}

BN-PAGE was carried out according to Schägger and von Jagow [31]. A 4- $\mu$ l aliquot of purified mitochondrial fraction (corresponding to $100 \mu \mathrm{g}$ protein) was solubilized with $40 \mu \mathrm{l}$ of a solution containing $0.5 \% \mathrm{n}$-dodecyl- $\beta$-Dmaltoside in $750 \mathrm{mM}$ 6-aminocaproic acid/50 mM bistris [bis-(2-hydroxyethyl)-amino-tris(hydroxymethyl)methane] for $15 \mathrm{~min}$ on ice. The solution was centrifuged at $18,000 \times g$ for $30 \mathrm{~min}$ at $4^{\circ} \mathrm{C}$ in order to sediment insoluble material. The supernatant was supplemented with $1.5 \mathrm{\mu l} 5 \%$ Coomassie Brilliant Blue G250 in $750 \mathrm{mM} \mathrm{6-}$ aminocaproic acid/50 mM bis-tris and $5.0 \mu \mathrm{l} 50 \%$ (v:v) glycerol. Samples were loaded onto a $1.5-\mathrm{mm}$ thick, 4$12 \%$ polyacrylamide linear gradient gel without a stacking gel and electrophoresed for $2 \mathrm{hr}$ at $150 \mathrm{~V}, 2 \mathrm{hr}$ at $350 \mathrm{~V}$ and $\sim 5 \mathrm{hr}$ at $500 \mathrm{~V}$ at $4^{\circ} \mathrm{C}$. Gels were either a) stained with a solution of 0.025\% Coomassie Brilliant Blue R250, 40\% ethanol, $10 \%$ acetic acid or b) gel lanes were excised for in-gel enzyme activity assays or two-dimensional BN/ SDS-PAGE. Molecular weights were estimated using the NativeMark unstained protein standard (Invitrogen).

\section{In-gel Enzyme Activity Assay}

In order to identify enzymatically active CI, in-gel activity assays were performed. A lane from a BN-PAGE experiment was excised and incubated overnight in $5 \mathrm{ml} 50 \mathrm{mM}$ MOPS.NaOH, pH 7.4 buffer containing $0.1 \mathrm{mg} / \mathrm{ml}$ reduced $\beta-\mathrm{NADH}$ and $1 \mathrm{mg} / \mathrm{ml}$ nitroblue tetrazolium. Active CI was identified by the formation of a purple-blue formazan precipitate at approximately $940 \mathrm{kDa}$.

\section{Two-dimensional BN/SDS-PAGE}

After one-dimensional BN-PAGE, a gel strip was excised and treated for $45 \mathrm{~min}$ with $10 \mathrm{ml}$ of a $0.125 \mathrm{M}$ Tris. $\mathrm{HCl}$ buffer, pH 6.8 ( $1 \times$ SDS-PAGE stacking buffer), containing $2 \%$ sodium dodecyl sulfate (SDS) and $1 \% \beta$-mercaptoethanol. The strip was incubated for $10 \mathrm{~min}$ in $10 \mathrm{ml}$ of the same solution, minus $\beta$-mercaptoethanol (a potent inhibitor of polymerization). The BN gel strip was encased in $4 \%$ SDS-PAGE stacking gel poured on top of a $15 \%$ resolving gel. Electrophoresis was carried out at room temperature at a constant current of $30 \mathrm{~mA}$ for approxi- 
mately 6 hr. The gel was stained with the BioRad Silver Stain Plus kit according to manufacturer's protocols.

\section{Reversed-phase HPLC and MS/MS}

The enzymatically active $\mathrm{CI}$ and inactive $\mathrm{CI}$ complexes were excised and each was placed in a separate microcentrifuge tube. The proteins were reduced with $10 \mathrm{mM}$ dithiothreitol in $100 \mathrm{mM}$ ammonium bicarbonate (AB) for $30 \mathrm{~min}$ at $56^{\circ} \mathrm{C}$ and alkylated with $100 \mathrm{mM}$ iodoacetamide in $100 \mathrm{mM} \mathrm{AB}$ for $30 \mathrm{~min}$ in the dark at room temperature. Proteins were incubated with trypsin overnight $(\sim 16 \mathrm{hr})$ at $37^{\circ} \mathrm{C}$ with $12.5 \mathrm{ng} / \mu \mathrm{l}$ modified porcine trypsin (Promega) in $100 \mathrm{mM} \mathrm{AB}$. Peptides were extracted once with $100 \mathrm{mM} \mathrm{AB}$ and twice with a 50:50 (v:v) acetonitrile: $\mathrm{H}_{2} \mathrm{O}$ solution containing $0.2 \%$ formic acid. All samples were analyzed by LC-MS/MS using an Agilent 1100 HPLC system equipped with a $15 \mathrm{~cm} \times 100 \mathrm{~mm}$ Onyx Monolythic $\mathrm{C}_{18}$ column (Phenomenex, Torrance, California). The separation was carried out using the following gradient: $2 \% \mathrm{~B}$ for $3 \mathrm{~min}$ increasing to $25 \% \mathrm{~B}$ over $45 \mathrm{~min}$ and $95 \% \mathrm{~B}$ over $10 \mathrm{~min}$ (A: $0.1 \%$ formic acid in water; B: $0.1 \%$ formic acid in acetonitrile) at $2 \mu \mathrm{l} / \mathrm{min}$. The HPLC was interfaced to an AB/MDS-SCIEX QTrap 4000 mass spectrometer via a nanoflow source. Data were acquired in the information-dependent acquisition mode, i.e., the $\mathrm{m} / \mathrm{z}$ values of the tryptic peptides were measured using an MS scan, followed by enhanced resolution scans of the three most intense peaks and finally three tandem MS scans. The tandem MS spectra were submitted to the database search program MASCOT [32] in order to identify the proteins. Data files were searched against $A$. castellanii EST clusters from TBestDB [20], a collection of Acanthamoeba ESTs generated by 454 pyrosequencing (kindly made available by BJ Loftus, University College Dublin), the current nuclear genome assembly (A. castellanii genome project), and the $A$. castellanii mitochondrial genome sequence [33]. The current $A$. castellanii genome assembly and Loftus EST data set are both publicly available from the Human Genome Sequencing Center, Baylor College of Medicine http://www.hgsc. bcm.tmc.edu/projects/microbial/microbial-pubPreview. xsp?project_id=163.

\section{Homology searches, alignments, mTP prediction}

The amino acid sequence of Arabidopsis thaliana $\gamma \mathrm{CA} 1$ (AT1G5980) was used to query the nr protein database at NCBI with BLASTp, as well as EST and nuclear genome databases from a wide variety of eukaryotes with tBLASTn [34]. Nucleic acid sequences were translated using the transeq program of the EMBOSS package and protein sequences were inferred manually. Protein homologs were aligned with Muscle v3.6 [35] using default parameters and edited with the BioEdit Sequence Alignment Editor.
TargetP [24] and MitoProt II [25] were used to assess the probability of mitochondrial localization for eukaryotic $\gamma$ CA homologs. When TargetP was used, the 'Plant' organism group was selected for organisms with primary or secondary plastids in order to include the possibility of plastid-targeted $\gamma C A$ proteins; for species lacking a plastid, the 'Animal' organism group was selected. MitoProt II does not have an option for assessing plastid localization.

\section{Phylogenetic analysis}

The AcCa1 protein sequence was used to query the $\mathrm{nr}$ database at NCBI via BLASTp. The best scoring homologs (i.e., ones having the lowest $\mathrm{E}$ - values) from a variety of major bacterial groups were retrieved and aligned with eukaryotic $\gamma$-type CA homologs using Muscle v.3.6 [35]. The alignment was edited manually with eBioX and was then used to reconstruct the maximum likelihood phylogenetic tree using RAxML-HPC [36] under the WAG $+\Gamma$ model (using the PROTGAMMAWAGF option) with 25 categories of substitution rate variation. One hundred bootstrap replicates were performed as a measure of statistical support for inferred nodes.

\section{List of abbreviations}

CI: complex I of the respiratory chain; $\gamma C A$ : $\gamma$-type carbonic anhydrase; Cam: archetypal $\gamma \mathrm{CA}$ from Methanosarcina thermophila; AcCa1 and AcCa2: names given to $\gamma$-type carbonic anhydrases from Acanthamoeba; NADH: nicotinamide adenine dinucleotide (reduced); BN-PAGE: blue native polyacrylamide gel electrophoresis; mTP: mitochondrial targeting peptide; EST: expressed sequence tag; ETC: electron transport chain

\section{Additional material}

\footnotetext{
Additional File 1 Table of $\mathrm{Cl}$ and $\mathrm{Cl}^{*}$ proteins identified by tandem mass spectrometry. The table lists proteins identified in the $940 \mathrm{kDa}(\mathrm{Cl})$ and $820 \mathrm{kDa}\left(\mathrm{Cl}^{*}\right)$ complexes via MASCOT searches of a six-frame translation of a 454 EST database $(\mathrm{N})$ and predicted proteins from the Acanthamoeba mitochondrial genome (M). Proteins with ion scores of 37 and above are reported from the 454 EST database while ion scores of 29 or higher are reported for inferred mtDNA-encoded proteins. Bolded entries highlighted in color indicate that the protein in question was identified in both $\mathrm{Cl}$ and $\mathrm{Cl}^{*}$ complexes. The two categories designated 'Other Proteins' are assumed to be contaminants; in particular, CV components (ATP synthase subunits) appear to be exceptionally abundant mitochondrial proteins, as judged by the results of MS/MS analysis of whole mitochondria.

Additional File 2 MS/MS peptide coverage. The peptides identified by MS/MS analysis in AcCa1 and AcCa2 are mapped onto the corresponding protein sequences. Each peptide is colour-coded according to whether it was identified only in the $940 \mathrm{kDa} \mathrm{Cl}$, only the $820 \mathrm{kDa} \mathrm{Cl}^{*}$, or in both samples.

Additional File 3 Sequences of $\mathbf{\gamma C A}$ proteins. The inferred protein sequences of $\gamma C A$ proteins are reported in fasta format. Proteins are designated as indicated in Additional File 5.

Additional File 4 Phylogenetically broad alignment of eukaryotic and prokaryotic $\gamma$ CAs.: A multiple alignment of eukaryotic and prokaryotic $\gamma C A s$ including complete and partial sequences from diverse eukaryotic groups.
} 
Additional File 5 Table of accession numbers. The table compiles acces sion numbers and other relevant information for $\mathrm{YCA}$ homologs available in public databases.

\section{Additional File 6 Table of mitochondrial targeting probabilities. The} table reports the probabilities of mitochondrial localization for $\mathrm{YCA}$ proteins (according to TargetP and MitoProtII). Bolded entries indicate proteins predicted to be mitochondrial with $\geq 70 \%$ probability.

\section{Authors' contributions}

RMRG performed the mitochondrial isolations, BN-PAGE, two-dimensional BN/ SDS-PAGE and sample preparation for MS/MS. RMRG performed bioinformatic analyses. RMRG and MWG prepared the manuscript. Both authors read and approved the final manuscript.

\section{Acknowledgements}

RMRG was supported by a Canada Graduate Scholarship (Doctoral) from the Natural Sciences and Engineering Research Council (NSERC) and a Predoctoral Scholarship from the Killam Trusts. This work was supported by operating grant MOP-4124 to MWG from the Canadian Institutes of Health Research. The authors thank DM Pinto and KA Chisholm (National Research Council Canada, Institute for Marine Biosciences, Halifax, NS) for assistance with mass spectrometry.

\section{Author Details}

Centre for Comparative Genomics and Evolutionary Bioinformatics, Department of Biochemistry and Molecular Biology, Dalhousie University, Halifax, Nova Scotia B3H 1X5, Canada

Received: 20 April 2010 Accepted: 14 June 2010

Published: 14 June 2010

\section{References}

1. Brandt U: Energy converting NADH:quinone oxidoreductase (complex I). Annu Rev Biochem 2006, 75:69-92

2. Weidner U, Geier S, Ptock A, Friedrich T, Leif H, Weiss $H$ : The gene locus of the proton-translocating NADH: ubiquinone oxidoreductase in Escherichia coli. Organization of the 14 genes and relationship between the derived proteins and subunits of mitochondrial complex I. J Mol Biol 1993, 233:109-122.

3. Carroll J, Fearnley IM, Skehel JM, Shannon RJ, Hirst J, Walker JE: Bovine complex I is a complex of 45 different subunits. J Bio/ Chem 2006, 281:32724-32727.

4. Abdrakhmanova A, Zickermann V, Bostina M, Radermacher M, Schägger $\mathrm{H}$, Kerscher S, Brandt U: Subunit composition of mitochondrial complex I from the yeast Yarrowia lipolytica. Biochim Biophys Acta 2004, 1658:148-156.

5. Marques I, Duarte M, Assuncao J, Ushakova AV, Videira A: Composition of complex I from Neurospora crassa and disruption of two "accessory" subunits. Biochim Biophys Acta 2005, 1707:211-220.

6. Cardol P, Vanrobaeys F, Devreese B, Van Beeumen J, Matagne RF, Remacle C: Higher plant-like subunit composition of mitochondrial complex I from Chlamydomonas reinhardtii: 31 conserved components among eukaryotes. Biochim Biophys Acta 2004, 1658:212-224.

7. Heazlewood JL, Howell KA, Millar AH: Mitochondrial complex I from Arabidopsis and rice: orthologs of mammalian and fungal components coupled with plant-specific subunits. Biochim Biophys Acta 2003, 1604:159-169.

8. Gray MW, Burger G, Lang BF: Mitochondrial evolution. Science 1999, 283:1476-1481.

9. Gabaldón T, Rainey D, Huynen MA: Tracing the evolution of a large protein complex in the eukaryotes, NADH:ubiquinone oxidoreductase (Complex I). J Mol Biol 2005, 348:857-870.

10. Parisi G, Perales M, Fornasari MS, Colaneri A, Gonzalez-Schain N, GomezCasati D, Zimmermann S, Brennicke A, Araya A, Ferry JG, Echave J, Zabaleta E: Gamma carbonic anhydrases in plant mitochondria. Plant Mol Biol 2004, 55:193-207.

11. Alber BE, Ferry JG: A carbonic anhydrase from the archaeon Methanosarcina thermophila. Proc Natl Acad Sci USA 1994, 91:6909-6913.

12. Ferry JG: The gamma class of carbonic anhydrases. Biochim Biophys Acta 2009, 1804:374-381.
13. Dudkina NV, Eubel H, Keegstra W, Boekema EJ, Braun HP: Structure of a mitochondrial supercomplex formed by respiratory-chain complexes I and III. Proc Natl Acad Sci USA 2005, 102:3225-3229.

14. Sunderhaus S, Dudkina NV, Jansch L, Klodmann J, Heinemeyer J, Perales M, Zabaleta E, Boekema EJ, Braun HP: Carbonic anhydrase subunits form a matrix-exposed domain attached to the membrane arm of mitochondrial complex I in plants. J Biol Chem 2006, 281:6482-6488.

15. Perales $M$, Eubel $H$, Heinemeyer J, Colaneri A, Zabaleta E, Braun HP: Disruption of a nuclear gene encoding a mitochondrial gamma carbonic anhydrase reduces complex I and supercomplex I + III2 levels and alters mitochondrial physiology in Arabidopsis. J Mol Biol 2005, 350:263-277.

16. Martin V, Villarreal F, Miras I, Navaza A, Haouz A, Gonzalez-Lebrero RM, Kaufman SB, Zabaleta E: Recombinant plant gamma carbonic anhydrase homotrimers bind inorganic carbon. FEBS Lett 2009, 583:3425-3430.

17. Braun $\mathrm{H}$, Zabaleta E: Carbonic anhydrase subunits of the mitochondrial NADH dehydrogenase complex (complex I) in plants. Physiol Plantarum 2007, 129:114-122.

18. Keeling PJ, Burger G, Durnford DG, Lang BF, Lee RW, Pearlman RE, Roger AJ, Gray MW: The tree of eukaryotes. Trends Ecol Evol 2005, 20:670-676.

19. Navet $R$, Jarmuszkiewicz W, Douette P, Sluse-Goffart CM, Sluse FE: Mitochondrial respiratory chain complex patterns from Acanthamoeba castellanii and Lycopersicon esculentum: comparative analysis by BNPAGE and evidence of protein-protein interaction between alternative oxidase and complex III. J Bioenerg Biomembr 2004, 36:471-479.

20. O'Brien EA, Koski LB, Zhang Y, Yang L, Wang E, Gray MW, Burger G, Lang BF: TBestDB: a taxonomically broad database of expressed sequence tags (ESTs). Nucleic Acids Res 2007, 35:D445-D451.

21. Soto AR, Zheng H, Shoemaker D, Rodriguez J, Read BA, Wahlund TM: Identification and preliminary characterization of two cDNAs encoding unique carbonic anhydrases from the marine alga Emiliania huxleyi. Appl Environ Microbiol 2006, 72:5500-5511.

22. Smith DG, Gawryluk RM, Spencer DF, Pearlman RE, Siu KW, Gray MW Exploring the mitochondrial proteome of the ciliate protozoon Tetrahymena thermophila: direct analysis by tandem mass spectrometry. J Mol Biol 2007, 374:837-863.

23. Neupert W, Herrmann JM: Translocation of proteins into mitochondria. Annu Rev Biochem 2007, 76:723-749.

24. Emanuelsson O, Nielsen H, Brunak S, von Heijne G: Predicting subcellular localization of proteins based on their $\mathrm{N}$-terminal amino acid sequence. J Mol Biol 2000, 300:1005-1016.

25. Claros MG, Vincens P: Computational method to predict mitochondrially imported proteins and their targeting sequences. Eur J Biochem 1996, 241:779-786.

26. Loftus B, Anderson I, Davies R, Alsmark UC, Samuelson J, Amedeo P, Roncaglia P, Berriman M, Hirt RP, Mann BJ, Nozaki T, Suh B, Pop M, Duchene M, Ackers J, Tannich E, Leippe M, Hofer M, Bruchhaus I, Willhoeft U, Bhattacharya A, Chillingworth T, Churcher C, Hance Z, Harris B, Harris D, Jagels K, Moule S, Mungall K, Ormond D, Squares R, Whitehead S, Quail MA, Rabbinowitsch E, Norbertczak H, Price C, Wang Z, Guillen N, Gilchrist C, Stroup SE, Bhattacharya S, Lohia A, Foster PG, Sicheritz-Ponten T, Weber C, Singh U, Mukherjee C, El-Sayed NM, Petri WA, Clark CG, Embley TM, Barrell B, Fraser CM, Hall N: The genome of the protist parasite Entamoeba histolytica. Nature 2005, 433:865-868.

27. Perales M, Parisi G, Fornasari MS, Colaneri A, Villarreal F, Gonzalez-Schain N, Echave J, Gomez-Casati D, Braun HP, Araya A, Zabaleta E: Gamma carbonic anhydrase like complex interact with plant mitochondrial complex I. Plant Mol Biol 2004, 56:947-957.

28. Zimmerman SA, Ferry JG: Proposal for a hydrogen bond network in the active site of the prototypic gamma-class carbonic anhydrase. Biochemistry 2006, 45:5149-5157

29. Tripp BC, Bell CB, Cruz F, Krebs C, Ferry JG: A role for iron in an ancient carbonic anhydrase. J Biol Chem 2004, 279:6683-6687.

30. Lohan AJ, Gray MW: Analysis of 5'- or 3'-terminal tRNA editing: mitochondrial 5' tRNA editing in Acanthamoeba castellanii as the exemplar. Methods Enzymol 2007, 424:223-242.

31. Schägger $H$, von Jagow $G$ : Blue native electrophoresis for isolation of membrane protein complexes in enzymatically active form. Anal Biochem 1991, 199:223-231. 
32. Perkins DN, Pappin DJ, Creasy DM, Cottrell JS: Probability-based protein identification by searching sequence databases using mass spectrometry data. Electrophoresis 1999, 20:3551-3567.

33. Burger G, Plante I, Lonergan KM, Gray MW: The mitochondrial DNA of the amoeboid protozoon, Acanthamoeba castellanii: complete sequence, gene content and genome organization. J Mol Biol 1995, 245:522-537.

34. Altschul SF, Madden TL, Schaffer AA, Zhang J, Zhang Z, Miller W, Lipman DJ: Gapped BLAST and PSI-BLAST: a new generation of protein database search programs. Nucleic Acids Res 1997, 25:3389-3402.

35. Edgar RC: MUSCLE: a multiple sequence alignment method with reduced time and space complexity. BMC Bioinformatics 2004, 5:113

36. Stamatakis A: RAxML-VI-HPC: maximum likelihood-based phylogenetic analyses with thousands of taxa and mixed models. Bioinformatics 2006, 22:2688-2690

doi: 10.1186/1471-2148-10-176

Cite this article as: Gawryluk and Gray, Evidence for an early evolutionary emergence of ?-type carbonic anhydrases as components of mitochondrial respiratory complex I BMC Evolutionary Biology 2010, 10:176

Submit your next manuscript to BioMed Central and take full advantage of:

- Convenient online submission

- Thorough peer review

- No space constraints or color figure charges

- Immediate publication on acceptance

- Inclusion in PubMed, CAS, Scopus and Google Scholar

- Research which is freely available for redistribution

Submit your manuscript at www.biomedcentral.com/submit
C) Biomed Central 